\title{
Die Forschungsgruppe Ethisch-Ökologisches Rating (FG EÖR) am Fachbereich Katholische Theologie der Goethe-Universität Frankfurt
}

\author{
Johannes J. Hoffmann
}

\section{Zusammenfassung}

Der Beitrag informiert über die Arbeit der Forschungsgruppe EthischÖkologisches Rating der Goethe-Universität in Frankfurt. Die Gruppe war interdisziplinär, interkulturell, ökumenisch und transdisziplinär zusammengesetzt. Sie hat als erste bereits in den 90-er Jahren anhand einer Wertbaumanalyse eine wissenschaftlich gestützte Kriteriologie zur Bewertung von Kapitalanlagen, den sogenannten Frankfurt-Hohenheim Leitfaden (FHL) entwickelt. Der FHL wurde gemeinsam mit einer Ratingagentur, nämlich der oekom research $\mathrm{GmbH}$ in München, in ein Ratingkonzept übertragen, nämlich das Corporate Responsibility Rating (CRR). Der Beitrag informiert über die Entwicklung, Anwendung, Grenzen und die mögliche Weiterentwicklung des Konzeptes. Inzwischen wird das CRR weltweit umgesetzt.

\section{Die Forschungsgruppe Ethisch-Ökologisches Rating}

Die FG EÖR wird seit nunmehr 25 Jahren durch das gemeinsame Interesse an nachhaltiger Entwicklung im Rahmen der Marktwirtschaft motiviert. Unser Beitrag ist getragen von dem Bemühen, verborgene Sachverhalte bloßzulegen, Ungesehenes sichtbar zu machen, aus überholten Traditionen herauszulocken, Mut für neue Wege zu machen, effektive Altruisten zu begleiten und zu fördern,

\section{J. J. Hoffmann ( $\varangle)$}

Arbeitsgruppe Ethisch-Ökologisches Rating, Institut für Katholische Theologie der Goethe-Universität in Frankfurt am Main, Frankfurt am Main, Deutschland E-Mail: j.hoffmann@em.uni-frankfurt.de 
damit Menschwerdung in Gemeinschaft im Mit-Sein mit der Schöpfung gelingen kann.

Nach über 25 Jahren wissenschaftlicher Arbeit der FG EÖR zur Entwicklung von ökologischer, sozialer, ökonomischer und interkultureller Nachhaltigkeit in der Marktwirtschaft ist es durchaus sinnvoll, einmal an die Anfänge zu erinnern und einen Blick auf die gegenwärtig laufende Arbeit zu werfen, um daraus Überlegungen für die zukünftige Forschungs- und Bewusstseinsbildungsarbeit zu gewinnen.

Zwei Grundfragen haben uns von Anfang an bewegt:

a) Welche Bedeutung hat die Allgemeine Menschenrechtserklärung für die Realisierung menschenwürdiger Lebensbedingungen in unserer und in uns fremden Kulturen?

b) Welche Konsequenzen ergeben sich daraus für die Gestaltung einer Marktwirtschaft, dass durch sie die Erhaltung der Substanz ökonomischer, ökologischer, sozialer und kulturelle Ressourcen für uns und künftige Generationen gesichert werden kann?

Erkenntnisleitend waren für uns dabei immer zwei Grundsätze. Einerseits waren wir davon überzeugt, dass wir nur kleinschrittige Veränderungen des Normalbereichs anstreben können, weil nach unserer Überzeugung nur so tragfähige Veränderungen in Wirtschaft und Gesellschaft erreichbar sind. In dieser Auffassung sahen wir uns von der Evolutionsbiologie bestärkt, die davon ausgeht, dass nur kleinschrittige Veränderungen des Normalbereichs Evolution bedeutet. Große Sprünge im Sinne von Mutationen sind nicht lebensfähig.

Der zweite erkenntnisleitende Grundsatz unserer Forschungsarbeit war immer und ist es auch jetzt, dass wir unsere Vorschläge im Dialog mit allen Stakeholdern in der Gesellschaft und in den betroffenen Kulturen erarbeiten müssen entsprechend der soziologischen Erfahrung, dass alle Systeme, Mechanismen, gesellschaftlichen Formen und kulturelles internalisiertes Ordnungswissen Ergebnisse sozialer Prozesse in Kulturen sind, die daher auch nur über soziale Prozesse in Kulturen verändert werden können.

In der FG EÖR arbeiten Frauen und Männer aus Wissenschaft und Praxis in einer flachen Hierarchie unter der Leitung des Sozialethikers Prof. Dr. Johannes Hoffmann und dem Wirtschaftswissenschaftler Prof. Dr. Gerhard Scherhorn zusammen. Seit einigen Jahren gibt es ein erweitertes Leitungsteam, das für die Bereiche „Wissenschaft“, „Wirtschaft“, „Politik“ und „Zivilgesellschaft“ zuständig ist. Die FG EÖR ist von Anfang an interdisziplinär, ökumenisch, interkulturell und transdisziplinär zusammengesetzt (Vgl. Borgwardt 2017). Anlässlich der 
Präsentation der Umsetzung des Frankfurt- Hohenheimer Leitfadens (FHL) im Jahr 2000 hob Prof. Dr. Rudolf Steinberg, der damalige Präsident der GoetheUniversität, als besonderes Merkmal die praxisorientierte Arbeit der FG EÖR hervor:

\begin{abstract}
„...weil in diesem Projekt in vielerlei Hinsicht sozusagen auch das Selbstverständnis der Goethe-Universität sichtbar wird. Dieses wird bei Ihrem Projekt in dreifacher Weise deutlich:
\end{abstract}

Zum ersten beleuchtet es das Problem des sogenannten Praxisbezugs universitärer Wissenschaft ... Aber Universitäten sind eben nicht nur der unberührte Hort der Wissenschaft, sondern immer auch Teil der Gesellschaft, mit der sie deren Probleme und Hoffnungen teilen. Und daher sind sie immer auch Orte, an denen Fragestellungen aus der Gesellschaft für die Gesellschaft bearbeitet werden ... das Projekt EthischÖkologisches Rating ist in diesem Sinne ein beeindruckendes Beispiel universitärer Wissenschaft. Es ist aber - zweitens - auch ein schönes Modell, was den sogenannten Wissenstransfer angeht ... Hier wurde nicht ansatzweise bloß Wissen von der Universität nach draußen transferiert, der Transfer erfolgte in beide Richtungen. Der entscheidende Punkt in dem Ganzen war aber wohl, dass sich die universitäre Seite nicht damit begnügte, ihren wissenschaftlichen Beitrag zu liefern und damit die Sache für sich als abgeschlossen betrachtete, sondern weiter die Umsetzung ihrer Ergebnisse begleitete ... Nicht nur wegen seines Themas, sondern auch wegen dieser besonderen Verantwortlichkeit, dieser Wissenschaftsethik selbst, scheint mir das Projekt EthischÖkologisches Rating vorbildlich.

Und schließlich noch ein Wort zu unserer Tradition. Als Wilhelm Merton, einer unserer Gründungsväter, sein Institut für Gemeinwohl ins Leben rief, wollte er seinen Mitmenschen, [Zitat] 'aus Elend, Laster und Unverstand' heraushelfen.

Damals, 1891, stellte sich das Problem ein wenig anders als heute. Die Industrialisierung und die Modernisierung hatten ihre sozialen Opfer, der Einzelne war stark gefordert, viele waren überfordert. Aber Mertons Idee der praktischen Hilfestellung wurde rasch durch die Einsicht ergänzt, dass man auch die Grundlagen der Modernisierung und ihrer Probleme untersuchen und theoretisch durchdringen müsse. Praktische Hilfe und Wissenschaft waren von Anfang an zusammen. An diesem Punkt kam zum Vorschein, was man gemeinhin die Verantwortung der Wissenschaft nennt."1

\footnotetext{
${ }^{1}$ Grußwort des Präsidenten Professor Dr. Rudolf Steinberg anlässlich der Präsentation der Forschungsergebnisse der Projektgruppe Ethisch-ökologisches Rating am Fachbereich Katholische Theologie in der Aula der Goethe- Universität am 15. September 2000.
} 


\section{Initiative für die Bildung der FG EÖR}

Grundlage waren die Bestimmungen des Grundgesetzes über die Sozialpflichtigkeit des Eigentums (Artikel 14, 2 GG), über den Schutz der natürlichen Lebensgrundlagen (Artikel 20a GG) und die Aussage in der Bayerischen Verfassung: „Alle wirtschaftliche Tätigkeit dient dem Gemeinwohl“ (Artikel 151). All diese Bestimmungen begründen aber noch kein subjektives Recht. Es muss gesetzlich geregelt werden. Dafür müssen wir etwas tun und uns engagieren.

Daher führte der Verfasser dieses Beitrags drei interkulturelle Symposien zum Thema „Das eine Menschenrecht für alle und die vielen Lebensformen“ durch, zu denen Fachleute aus allen Kontinenten eingeladen wurden. Die Symposien fanden in den Jahren 1989, 1990 und 1991 in der KfW in Frankfurt statt (vgl. Hoffmann 1991, 1994).

Schließlich kamen drei Manager der Deutschen Bank im Jahr 1990 zu Johannes Hoffmann mit der Frage, ob er bereit sei, mit ihnen über die ethische Bewertung von Kapitalanlagen zu sprechen mit der Begründung, dass die Kirchen bei der Deutschen Bank Kapitalanlagen in zweistelliger Milliardenhöhe angelegt hätten, dieselben aber gar nicht wüssten, was die Deutsche Bank mit den Geldern mache, ob nicht die Art der Anlage mehr Schaden anrichte als der Nutzen, den die Kirchen mit den Gewinnen daraus bewirken können. Die Gespräche mündeten in der Konzipierung einer Fachtagung, die im März 1991 in der Evangelischen Akademie Bad Boll zum Thema:

„Saubere Gewinne - Ethische Vermögensanlagen in der Diskussion“ stattfand (Roche et al. 1992). Als Ergebnis der Tagung kam die Anfrage an Johannes Hoffmann, ob er nicht eine Forschungsgruppe gründen könnte, die eine differenzierte methodisch gestützte Kriteriologie entwickeln sollte, die für Deutschland, das Land der Dichter und Denker, angemessen sei. Der Vorschlag wurde aufgegriffen und es kam zusammen mit Prof. Dr. Gerhard Scherhorn, Universität StuttgartHohenheim zur Bildung der Forschungsgruppe Ethisch-Ökologisches Rating noch im Jahr 1992. Im Rahmen des inhaltlichen Zuschnitts wurden in dieser Gruppe eine Reihe von Studien und Dissertationen erarbeitet, die als Grundlage und 
Ergänzung der Forschungsarbeit dienten. ${ }^{2}$ Ohne diese Arbeiten, die in Sitzungen der FG EÖR diskutiert und begleitet wurden, wären die Erkenntnisse und Ergebnisse der wissenschaftlichen Arbeit der Forschungsgruppe nicht zu denken.

Inzwischen gehören zu dieser Gruppe über 50 Mitglieder im In- und Ausland, von denen ein Kern von bis zu 15 Personen bei den Sitzungen der FG EÖR teilnimmt. Einige wirken korrespondierend mit.

${ }^{2}$ Armin SCHNEIDER: Ethik bei der Auswahl von Führungskräften, Frankfurt 1993; Roland MIERZWA: Die Konversionsbewegung im deutschen katholischen Raum: eine zeitgeschichtliche systematische Studie, Frankfurt 1998; Bernd Christian BALZ: Ethisch-ökologische Geldanlage - eine kapitalmarktorientierte Analyse, Frankfurt 1999; Claus F. LÜCKER: Zinsverbot und Schuldenerlass, Frankfurt 1999; Johannes HOFFMANN/Gwendolin WANDERER (Hg.): Ethische Implikationen veränderter Rahmenbedingungen in der sozialen Arbeit, Beispiel: Betreuungsrecht - Insolvenzrecht - Asylverfahren, Frankfurt 2000; Claudia DÖPFNER: Zur Glaubwürdigkeit ethisch-ökologischer Geld- und Kapitalanlagen- Eine theologisch-ethische Untersuchung auf dem Hintergrund der Frage nach der Glaubwürdigkeit der ökonomischen und monetären Strukturen, Frankfurt 2000; Peter GRIEBLE: Ethischökologische Geldanlage. Einflussmöglichkeiten durch Beachtung von ethisch-ökologischen Gesichtspunkten bei der Anlage von Geld, Frankfurt 2001; Hans-Albert SCHNEIDER: Ethisches Rating - Begründung, Bewertungsmöglichkeit, Evaluation, Frankfurt 2001; Lucia A. REISCH: Ethical-ecological Investment: Towards Global Sustainable Development, Frankfurt 2001; John Chidi NWAVOR:, Church and State: The Nigerian Experience, Frankfurt 2002; Joseph Okechukwu OFFOR: Community Radio and its Influence in the Society: The Case of Enugu State - Nigeria, Frankfurt 2002; Franziska JAHN: Zur Qualität von Nachhaltigkeitsratings. Zwischen Anspruch und Wirklichkeit, Frankfurt/London 2004; Claudia DÖPFNER: Kunst und Kultur - voll im Geschäft? Kulturverträgliches Kunstsponsering, Frankfurt/London 2004; HyunJu SHIM: Die Herausforderung der koreanischen Kultur durch die hegemoniale Globalisierung. Ein Beitrag zur Bestimmung des Verhältnisses intra-, supra- und transkultureller Werte, Frankfurt/London 2004; Armin SCHNEIDER: Wege zur verantwortlichen Organisation. Die Bedeutung der ethischen und theologischen Perspektive für die Qualität der Organisations- und Personalentwicklung, Frankfurt/London 2005; Clara E. LAEIS: Corporate Citi- zenship. Unternehmerische Bürgerkompetenz im Dienste der Erneuerung der Sozialen Marktwirtschaft. Ein Mittelstandskonzept, Münster 2005; Klaus GAB- RIEL: Nachhaltigkeitsindizes. Indices of Sustainability, Frankfurt 2005; Peter Okechukwu NWANKWO: Social Development in Rural Communities in South- Eastern Nigeria. A Mission of Charity, Frankfurt-London 2006; Ndidi Nnoli EDOZIEN: Ownership and Management Structures in the Economy. African Traditionel Values applied to Modern Issues of Sustainability and the Corporate Governance Function, Enugu 2007; Klaus GABRIEL: Nachhaltigkeit am Finanzmarkt. Mit ökologisch und sozial verantwortlichen Geldanlagen die Wirtschaft gestalten, München 2007; Chidi Leonhard ILECHUKWU: IGBO. Indigenious Economy and the Search for Sustainable Development in Post Colonial African Society. A Socio-Ethical Study, Enugu 


\section{Erkenntnisleitende Ideen}

Die kapitalistische Marktwirtschaft ist nach Ansicht der FG EÖR nicht zukunftsfähig, weil der Primat des Kapitals in einem sich verschärfenden Widerspruch zum marktwirtschaftlichen Prinzip steht.

Die Privilegierung des Finanzkapitals seit den 1970er Jahren hat diesen Widerspruch auf die Spitze getrieben. Er muss beseitigt werden, weil er die nachhaltige Entwicklung verhindert. Gesellschaftliche Fehlentwicklungen wie diese sind -soziologisch betrachtet- Ergebnisse sozialer Prozesse in Gesellschaften und Kulturen. Sie können - wie bereits angedeutet- daher soziologisch gesehen wieder durch neue soziale Prozesse geändert werden, wenn sie als Bedrohungen unseres Planeten wahrgenommen werden.

Gerade aus diesem Grund waren wir der Auffassung, dass radikale Umwälzungen nicht erfolgreich sein können. Es geht um kleinschrittige Veränderungen des Normalbereichs, da es sich um die Neujustierung eines komplexen Weltsystems handelt, das sich über Jahrhunderte hin entwickelt hat und auch durch Revolutionen nur in einzelnen seiner Elemente verändert wurde.

Daher setzen wir uns für die Änderung eines zentralen Elements ein: Die historische Vorrangstellung des Kapitals ist überholt, sie muss in einen Gleichrang der Produktivkräfte Natur, Arbeit und Kapital überführt werden, wie er für nachhaltige Entwicklung konstitutiv ist. Dazu müssen soziale Prozesse in Gang kommen, damit ein kultureller Druck entsteht, der die nötigen kleinschrittigen Veränderungen des gesellschaftlichen, kulturellen und ökonomischen Normalbereichs zu befördern vermag.

\section{$4 \quad$ Welche Hebel der Marktwirtschaft sind wir dabei angegangen?}

Ein erster Schritt war, der Absolutsetzung des Geldes durch die Entwicklung und Förderung des ethisch-ökologischen Investments entgegen zu treten. Georg Simmel hat dazu in seiner Philosophie des Geldes aus dem Jahr 1900 (Simmel 1989;

2008; Simeon RIES: Kulturverträgliches Management. Unternehmen zwischen Wettbewerb und kulturelle Verantwortung, Frankfurt 2008; Emanuel Franklyn Onyemaechi OGBUNWEZEH: Towards an ethical-ecological Assessment of Companies in Nigeria, Frankfurt etc. 2009; Sr. Veronika FRICKE osf: Nachhaltig investieren in Mikrofinanz?, Erkelenz 2011; Agi MAKIL: Nachhaltigkeit für Indien. Ethisch-ökologische Bewertung indischer Unternehmen auf Basis des Frankfurt-Hohenheimer Leitfadens und des Corporate Responsibility Ratings, 2011. 
vgl. auch Von Flotow 1995) angeregt, ethisch orientierte Investoren zu motivieren, Investition in ökologisch, sozial und kulturverträgliche Innovationen/Produktionen der Realwirtschaft zu realisieren, Unternehmer und Manager dazu zu ermutigen und dabei zu bestärken, ,die Wirtschaft entsprechend der Würde des Menschen und mit Blick auf das Gemeinwohl zu gestalten“3. In diesem Sinne haben wir Ermöglichungsbedingungen und Geltungsbedingungen erforscht.

$\mathrm{Zu}$ den Ermöglichungsbedingungen gehörte die Entwicklung einer Kriteriologie zur Bewertung von Unternehmen und Kapitalanlagen, das war der sog. Frankfurt-Hohenheimer Leitfaden (FHL), den wir mit der oekom research GmbH 1999 in ein Ratingkonzept übertrugen. Die oekom research $\mathrm{GmbH}$ machte dieses Ratingkonzept zu ihrem Hauptprodukt und erreichte damit als Nachhaltigkeitsagentur heute eine gewichtige Stellung am europäischen Markt und mauserte sich zur oekom research AG. In den folgenden Jahren eröffnete die Oekom research AG Büros in London, Paris und in New York. Am 29.3.2018 meldete die oekom research: „,oekom research wird Teil der Institutional Shareholder Services Inc., dem weltweit größten Anbieter von Corporate Governance und Responsible Investment-Lösungen. Um der Stärke und dem hohen Ansehen beider Marken Rechnung zu tragen, wird der so entstehende neue Geschäftsbereich ISS-oekom benannt."

\section{5 "Illusionen“ über die Wirkung von Nachhaltigkeitsratings}

Ziel des FHL war es, mit einer wissenschaftlichen Kriteriologie auch tatsächlich den Markt im Sinne der nachhaltigen Entwicklung (vgl. Rio-Erklärung 1992) ${ }^{4}$ zu beeinflussen. Deshalb hatten wir gehofft, dass eine spürbare Wirkung auf die Erhaltung und Verbesserung der sozial-ökologischen Marktwirtschaft ausgeht und das Erfordernis einer Kreislaufwirtschaft bewusst wird. Zwar steigt das Volumen nachhaltiger Geldanlagen kontinuierlich, dennoch ist die Welt heute im Großen und Ganzen keineswegs nachhaltiger als vor einem Vierteljahrhundert, d. h. die Wirkung die von Nachhaltigkeitsratings und auch von ethischen Geldanlagen ausgehen würde, ist von der Forschungsgruppe überschätzt worden.

\footnotetext{
${ }^{3}$ Kardinal Turkson am 18.9.12 in Frankfurt anlässlich der Präsentation der Handreichung des Päpstlichen Rates für Gerechtigkeit und Frieden

${ }^{4}$ Rio-Erklärung über Umwelt und Entwicklung. Die Konferenz der Vereinten Nationen über Umwelt und Entwicklung, zum Abschluss ihrer Tagung vom 3. bis 14. Juni 1992 in Rio de Janeiro
} 
Dies liegt nicht zuletzt an der Verwässerung des Nachhaltigkeitsbegriffs und des Nachhaltigkeitsverständnisses bei Nachhaltigkeitsagenturen und Investoren.

Märkte können nur so gut sein wie die Regeln, die sie befolgen; diese müssen von außen gesetzt und überwacht werden; dafür war es bisher noch zu früh. Denn wir haben es hier mit einem Markt zu tun, der sich neu entwickelt hat. Er scheint noch in der Experimentierphase zu sein. In diesem Stadium müssen erst einmal Kriterien gefunden und Erfahrungen gesammelt werden. Das können nur die Pionierunternehmen leisten, die als erste auf dem Markt tätig werden. Zwischen ihnen sind Unterschiede in den Maßstäben die Regel. So ist es kein Wunder, dass verschiedene Rating-Agenturen, die mit dem Anspruch auftreten, „Nachhaltigkeit“ zu bewerten, die Nachhaltigkeitsperformance einzelner Unternehmen unterschiedlich einschätzen. Man kann das kritisieren, muss aber zugleich den Unternehmen dankbar sein, dass sie das Neuland überhaupt beackern. Das ist schon für sich genommen ein großes Verdienst.

Mittlerweile nähern wir uns allerdings dem Zeitpunkt, an dem die Phase des Experimentierens als abgeschlossen betrachtet werden kann. Nach diesem Zeitpunkt sollten einheitliche Maßstäbe gelten. Das bedeutet, dass dann nicht so sehr die Professionalität und Transparenz der Untersuchungs- und Bewertungsverfahren verbessert werden muss; hier sind die Unterschiede zwar nennenswert, werden sich aber wohl noch abschleifen oder sind mit einigen Vorschriften relativ leicht zu glätten. Problematischer, weil grundsätzlich, ist die Differenz im Nachhaltigkeitsbegriff, den die Institute zugrunde legen.

Die einen steuern auf eine konsequent ethische Bewertung $\mathrm{zu}$, indem sie als nachhaltig die Erhaltung des Natur- und Sozialkapitals betrachten, also ein zugleich „ökologisch“ und „sozial“ nachhaltiges Wirtschaften fordern. Dazu müssen sowohl die naturgegebenen als auch die gesellschaftlich gestalteten Lebens- und Produktionsgrundlagen in ihrem Potenzial erhalten werden, das Naturkapital ebenso wie das Sozialkapital. Beide können nur aufrechterhalten werden, wenn die Unternehmen auf längere Sicht keine Verluste machen, sodass auch das reale Wirtschaftskapital, der Gesamtwert des privaten Produktiv- und Humankapitals, mindestens erhalten bleibt. Ökonomisch betrachtet läuft Nachhaltigkeit auf das Gleiche hinaus wie ökologisch und sozial betrachtet: Unter allen drei Blickwinkeln geht es um die Bewahrung der Substanz, der Lebensund Produktionsgrundlagen.

Die anderen weisen dem ,ökonomisch“ nachhaltigen Wirtschaften eine darüber hinausgehende, eigene Bedeutung zu, indem sie verlangen, dass nachhaltiges Wirtschaften über die Substanzerhaltung hinaus eine positive Rendite abwirft, also das Wirtschaftskapital steigert und sich somit auch „materiell“ lohnt. Damit interpretieren sie das Drei-Säulen-Modell auf eine Weise, die es in inneren 
Widerspruch bringt. Denn bisher gibt es keine Unternehmen, die vollständig nachhaltig wirtschaften. Selbst bei den am weitesten fortgeschrittenen beruht noch ein Teil des Gewinns darauf, dass sie irgendwelche Aufwendungen unterlassen (,externalisieren“), die zur Erhaltung der von ihnen genutzten Gemeinressourcen notwendig wären. Unterlassen werden z. B. Aufwendungen zur Vermeidung klimaschädlicher oder toxischer Emissionen, zur Wiedergewinnung bzw. zum Ersatz verbrauchter Rohstoffe, zur Regeneration beanspruchter Ökosysteme, zur Erhaltung des friedlich-kooperativen gesellschaftlichen Zusammenhalts. Wer Nachhaltigkeitsfortschritte von der Rendite abhängig macht, hat ein starkes Motiv, diejenigen Erhaltungsinvestitionen am längsten hinauszuschieben, deren Unterlassung bisher am meisten zum Gewinn beiträgt. Dem gleichen Verdacht sind Unternehmen ausgesetzt, die Wert darauf legen, nach einem an ihre individuellen Präferenzen angepassten (,,customized“) Bewertungsverfahren beurteilt zu werden.

Denn das Ziel der nachhaltigen Entwicklung besteht gemäß der Definition der Brundtland-Kommission und ihrer Interpretation durch die deutsche EnqueteKommission „Schutz des Menschen und der Umwelt“ darin, dass die genutzten Gemeinressourcen, seien sie naturgegeben oder gesellschaftlich gestaltet, nicht länger aufgezehrt, sondern in ihrem Potenzial für künftige Generationen so erhalten werden, dass diese in der Befriedigung ihrer Bedürfnisse nicht schlechter gestellt sind als die gegenwärtig Lebenden. An diesem Ziel muss auch jedes einzelne Unternehmen, jeder einzelne private oder öffentliche Haushalt gemessen werden.

Ein dieser Definition folgendes Nachhaltigkeitsrating darf das höchste Prädikat nur an Unternehmen vergeben, die alle genutzten Gemeinressourcen ebenso behandeln wie ihre eigenen Produktionsanlagen, indem sie jeden Verbrauch von Natur- und Sozialkapital durch geeignete Ersatzinvestitionen vermeiden oder kompensieren. Und die übrigen Stufen der Bewertungsskala müssen am Effekt der Erhaltungsinvestitionen orientiert sein: je größer die verbleibende Externalisierung, desto negativer die Bewertung. So würde nach und nach sichergestellt, dass die regenerierbaren Gemeinressourcen - die Ökosysteme, das Klimasystem, die menschliche Gesundheit, die gesellschaftliche Integration - sich regenerieren können und die nicht erneuerbaren Gemeinressourcen - verbrauchte Rohstoffe oder fossile Energiequellen - wiederverwendet oder durch erneuerbare ersetzt werden.

Gesamtwirtschaftlich muss das auf Dauer finanzierbar sein, doch die Nachhaltigkeitsbewertung des einzelnen Unternehmens darf keinesfalls, auch nicht zusätzlich, am Gewinn orientiert sein. Das verstößt gegen das Nachhaltigkeitsziel, das zwar ein Wachsen der nachhaltigeren, zugleich aber ein Schrumpfen der 
weniger nachhaltigen Produktionen fordert; und ebenso verletzt es das marktwirtschaftliche Prinzip, das die Marktleistung an realen Absatzsteigerungen misst und all jene Gewinne davon ausnimmt, die durch unlauteren Wettbewerb oder reine Finanzmanipulationen zustande kommen.

Wenn man also als Dimensionen der Nachhaltigen Entwicklung nicht nur die ökologische und die soziale, sondern auch die ökonomische betrachtet, so muss die Betrachtung an der realen "Substanz" orientiert sein, von der wir leben und die es zu erhalten gilt. Keine der drei Dimensionen ist durch eine andere substituierbar, schon gar nicht durch Finanzkapital. Dieses darf unter ihnen keine eigene Rolle spielen. Denn für die Kapitalrendite macht es keinen Unterschied, ob der Gewinn durch Erhaltung oder durch Aufzehrung der realen Substanz zustande kommt.

So muss die Weiterentwicklung des Nachhaltigkeitsratings den Unternehmen die Selbstkontrolle, aber auch die soziale Kontrolle darüber eröffnen, was und wie viel sie zur Erhaltung und Kultivierung der naturgegebenen, gesellschaftlich/kulturellen und realwirtschaftlichen Lebens- und Produktionsgrundlagen beitragen. Daran gibt es noch viel zu verbessern. Eine Entwicklungsaufgabe wird darin bestehen, die Bewertungskriterien stärker an der Erhaltung der Gemeinressourcen zu orientieren (Forschungsgruppe Ethisch Ökologisches Rating 2016). Eine andere gilt der Erweiterung der Bewertungspraxis auf die bisher ausgeklammerten Wirtschaftsbereiche, namentlich auf die Vergabe von Krediten, auf Finanztransaktionen jeder Art und nicht zuletzt auf die mittleren und kleinen Unternehmen.

Dieser Befund hat uns in einem zweiten Schritt zur Frage nach den Geltungsbedingungen - denn GG-Artikel sind nicht einklagbar - veranlasst und uns dem Wettbewerb als einem zentralen Hebel der Marktwirtschaft zugewandt.

- Externalisierung ist das Gegenteil von Nachhaltigkeit

- Nachhaltigkeit verlangt, dass die allgemeinen Lebensgrundlagen (BVerfG: Güter der Allgemeinheit) auch für künftige Generationen verfügbar bleiben.

- Nutzer müssen daher auch für die Erhaltung (Regeneration, Wiedergewinnung, ggf. Ersatz) sorgen.

Kaum jemand wird bestreiten, dass Marktwirtschaft und Wettbewerb untrennbar zusammengehören. Die Meinungen gehen allerdings aus-einander, wenn es um die Beurteilung der ethischen Qualität dieses Verhältnisses im Rahmen der Wirtschaft der Bundesrepublik im Kontext globaler und liberalisierter Bedingungen geht. Nicht zuletzt deswegen wurde vor nicht ganz 60 Jahren das Kartellgesetz 
erlassen und das Bundeskartellamt geschaffen. Anlässlich der Feier ,,50 Jahre Kartellgesetz“" wurde von Gerhard Hennemann mit Recht darauf hingewiesen:

\begin{abstract}
„Ohne Kartellverbot, Fusionskontrolle und Missbrauchsaufsicht, die fest im Gesetz gegen Wettbewerbsbeschränkungen (GWB) verankert sind, wäre die Sicherung effizienten Wettbewerbs heute sicherlich undenkbar, denn das Ordnungsprinzip des Wettbewerbs hat nun einmal keine Lobby. Vielmehr wird es durch einzelwirtschaftliche Interessen, die sich in Politik und Verbänden ihre Fürsprecher suchen, immer wieder neuen Belastungstests unterzogen.“(Hennemann 2008)
\end{abstract}

Ganz anders sehen das Karl Homann und Michael Ungethüm in ihrem Beitrag „Ethik des Wettbewerbs.“ Geradezu euphorisch schreiben sie dem „Wettbewerbsprinzip eine ethische Rechtfertigung“ zu und behaupten: „die Marktwirtschaft mit Wettbewerb ist das beste bisher bekannte System zur Verwirklichung der Solidarität aller Menschen unter modernen Bedingungen.“ Ferner: „Markt und Wettbewerb sind unter den Bedingungen moderner Großgesellschaften die effizienteste Form der Caritas, ... Markt und Wettbewerb können daher als institutionalisierte Form des Gebots der Nächstenliebe unter den Bedingungen moderner Großgesellschaften verstanden werden.“ (Homann und Ungethüm 2007)

Die verheerenden Verwüstungen unserer natürlichen, sozialen und kulturellen Lebensgrundlagen, die der Wettbewerb in der Marktwirtschaft angerichtet hat, sind auch nicht mit dem Verweis auf

Josef Schumpeters „Prozess der schöpferischen Zerstörung“ ethisch zu rechtfertigen.

\title{
6 Unsere Ausgangsthese aus sozialethischer Sicht
}

Wettbewerb und Marktwirtschaft müssen gemessen werden am Bei- trag zur Erreichung „universaler Solidarität in Freiheit als äußerster erreichbarer Idee“ (Peukert 1976) und am Substanzerhalt von Natur und Mitwelt zur Gewährleistung von Bioüberlebenssicherheit für alle Menschen. Oder anders ausgedrückt: Für die Ermöglichung einer Menschwerdung in Gemeinschaft im Mit-Sein mit der Schöpfung für alle Menschen.

Angesichts der absurden Entwicklung - die Finanzkrise und die Klimakatastrophe sind Beispiele -, in die nicht hinreichend geregelter Wettbewerb geführt hat, müssen die Gründe für Rahmenbedingungen sowie deren Inhalte zum Gegenstand der Wirtschaftstheorie und Wirtschaftspraxis gemacht werden. (Vgl. Scherhorn 2005, S. 136).

Fazit für die ethische Regulierung des Wettbewerbs 
Das Ergebnis der bisher angestellten Überlegungen lässt sich ethisch in sechs humanen Grundorientierungen zusammenfassen. (Vgl. Hoffmann et al. 1997)

a) Rücksicht und Fairness trotz Konkurrenz

b) Diskursbereitschaft statt Positionalität

c) Begrenzung partieller Interessen durch Respekt vor dem Gemeinwohl

d) Selbstbegrenzung im Wachstum

e) Kreativität mit Verantwortung

f) Verzicht auf das Recht des Stärkeren

Diese sechs ethischen Grundorientierungen sollten in den Rahmenbedingungen der Wettbewerbsgesetze ihren Niederschlag finden. Daraus würde ein innovatorischer nachhaltiger Wettbewerb resultieren, der eine zukunftsfähige Marktwirtschaft zur Folge hätte. Auf dem Hintergrund dieser ethischen Grundorientierung folgen nun Vorschläge für die Änderung der Wettbewerbsgesetze.

\section{$7 \quad$ Ethische Kritik des Wettbewerbsrechtes}

Das Wettbewerbsrecht aus einer ethischen Perspektive in den Blick zu nehmen, verlangt zunächst einmal, das Wettbewerbsrecht in seinen eigenen Zielsetzungen zur Kenntnis zu nehmen. Das ist im Rahmen dieses Beitrages nicht möglich, wurde aber vom Verfasser im Rahmen des interdisziplinär und interkulturell gestalteten Symposiums mit dem Titel: „Nachhaltigkeit als Gestaltungsprinzip für die Rahmenordnungen von Finanz- und Gütermärkten“ im Mai 2008 aufgezeigt. ${ }^{5}$ Im Schlusskommuniqué des Symposiums wurde festgehalten, ,wieweit das Wettbewerbsrecht im Hinblick auf nachhaltige Entwicklung verändert werden muss, wie Finanzmärkte zukunftsfähig werden und wie über das Konzept der Nachhaltigkeit so verschiedene Grundprobleme wie Klimawandel und Korruption überwunden werden können“ (Hoffmann 2009, S. 431).

Auf diesem Hintergrund schlägt die FG EÖR folgende Gesetzesänderungen vor, die in einem Appell an alle Wirtschaftssubjekte, an die Politiker und an die Abgeordneten des Deutschen Bundestages zusammengefasst wurden. In den Ausführungen haben die Überlegungen zu einer Ethik des Wettbewerbs und zur Kritik des Wettbewerbsrechtes Eingang gefunden.

\footnotetext{
5 Johannes HOFFMANN: Ethische Kritik des Wettbewerbsrechts, in: Johannes Hoffmann / Gerhard Scherhorn (Hg.): Eine Politik für Nachhaltigkeit. Neuordnung der Kapital- und Gütermärkte, Erkelenz 2009, 24-55.
} 
Hier der Wortlaut: Nachhaltige Entwicklung braucht Gesetze für nachhaltigen Wettbewerb.

Unsere Gesetze verhindern den Ressourcenschutz! Nachhaltiger Wettbewerb muss einklagbar werden!

- Die beliebige Verfügung über das Eigentum nach § 903 BGB

- muss unter den Vorbehalt gestellt werden, dass der Eigentümer die Kriterien der Natur- und Sozialverträglichkeit beachtet (Vgl. Hoffmann et al. 1997). Oder anders formuliert: „Der Eigentümer einer Sache kann, soweit nicht das Gesetz, Rechte Dritter oder zwingende Erfordernisse des Schutzes der natürlichen Gemeingüter oder der Volksgesundheit entgegenstehen, mit der Sache nach Belieben verfahren und andere von jeder Einwirkung ausschließen. “6

- Externalisierung muss in die verbotenen Wettbewerbshandlungen

- nach $\S 4$ des Gesetzes gegen den unlauteren Wettbewerb (UWG) aufgenommen werden, etwa durch einen zusätzlichen Absatz 12, in dem bestimmt wird, dass unlauter im Sinne von $\S 4$ handelt (und daher auch von einem Wettbewerber auf Unterlassung in Anspruch genommen werden kann), wer sich dadurch einen Wettbewerbsvorteil verschafft, dass er ,zwingende (oder auch anerkannte) Erfordernisse des Schutzes der natürlichen Gemein-güter oder der Volksgesundheit missachtet", und sich so Vorteile gegenüber denjenigen Mitbewerbern verschafft, die die natürlichen und sozialen Lebensgrundlagen schützen, indem sie diese Kosten selbst tragen. ${ }^{7}$ Das UWG soll ja verhindern, dass Unternehmen die Nachfrager durch bloß vorgespiegelte Leistungen für sich gewinnen. Ein durch Externalisierung von Kosten erreichter Preis- oder Qualitätsvorsprung ist in diesem Sinn nicht weniger unlauter und dem Allgemeinwohl nicht weniger abträglich als eine Täuschung der Nachfrager durch irreführende Werbung oder Ausnutzung von Unerfahrenheit. ${ }^{8}$

\footnotetext{
${ }^{6}$ Diese Formulierung hätte nach Prof. Dr. Thomas Raiser, Humboldtuniversität Berlin, den Vorteil, das Anliegen deutlich zum Ausdruck zu bringen und kann so auch zur Meinungsbildung im Volk und bei den politisch Verantwortlichen beitragen. Auf der anderen Seite führt sie, falls Zweifel an der Gemeinverträglichkeit eines bestimmten Eigentumsgebrauchs aufkommen oder darüber ein Streit entsteht, dazu, dass derjenige, der sich auf die Gemeinschädlichkeit beruft, darlegen muss, dass es wirklich zwingende Gründe sind, welche den angegriffenen Gebrauch des Eigentums untersagen.

${ }^{7}$ Eine entsprechende Definition der Externalisierung gehört auch in die „Schwarze Liste“ der Richtlinie 2005/29/EU über unlautere Geschäftspraktiken im Binnenmarktverkehr.

${ }^{8}$ Externalisierungsstrategien von Unternehmen könnten dann - etwa mit Hilfe der Zentralstelle zur Bekämpfung des unlauteren Wettbewerbs (www.wettbewerbszentrale.de) - von Mitbewerbern angeklagt werden, die sich durch diese Strategien benachteiligt fühlen und die
} 
- Flankierend müssen befristete Vereinbarungen zwischen Unternehmen, die die Internalisierung von bisher abgewälzten Kosten absichern, in $\S 7$ (1) Gesetzes gegen Wettbewerbsbeschränkungen (GWB) sowie Art. 81 (3) des EU-Vertrags vom Kartellverbot ausgenommen werden. Das GWB soll ja verhindern, dass Unternehmen ihren Gewinn dadurch steigern, dass sie Preisunterbietung oder Qualitätsüberbietung untereinander ausschalten. Es nimmt aber Vereinbarungen vom Kartellverbot aus, in denen Unternehmen Aufwendungen zur Verbesserung (z. B. Rationalisierung) der Produktion bzw. des Angebots verabreden. Eine Ausnahme muss auch für Verabredungen gelten, in denen Unternehmen sich darüber verständigen, bisher externalisierte Kosten künftig selbst zu tragen.

- § 93 AktG sollte um den Satz ergänzt werden: „Zu den Sorgfaltspflichten eines Vorstandsmitglieds gehört es auch, sich über zwingende Erfordernisse der Gemeinverträglichkeit seiner Entscheidungen oder ihrer Auswirkungen auf die Volksgesundheit hinreichend zu informieren und sie zu beachten." Der Unternehmensvorstand muss in $§ 76$ (1) des Aktiengesetzes (AktG) sowie Art. 4.1.1 des Deutschen Corporate Governance Kodex auch auf den Schutz der naturgegebenen und der gesellschaftlichen Gemeingüter verpflichtet werden, die unsere Lebens- und Produktionsgrundlagen bilden (des Natur- und Sozialkapitals). So bekommt der Vorstand gegenüber klagenden Aktionären eine Rechtsgrundlage für vertretbare Aufwendungen zugunsten des Umweltschutzes, der Arbeitsbedingungen oder der gesellschaftlichen Integration, und die Zivilgesellschaft gewinnt eine Chance, das Unternehmen daran zu erinnern, dass es auf nachhaltige Entwicklung verpflichtet ist.

- In das Kreditwesengesetz (KWG) und das Investmentgesetz (InvG) muss den Unternehmensleitern und Anlageberatern die Pflicht auferlegt werden, auch über bekannte gemeinschädliche oder gesundheitsschädliche Folgen der Produktionsmethoden, welche das die Auslagepapiere ausgebende Unternehmen anwendet, sowie über alternative Anlagemöglichkeiten zu informieren. Ferner sollten die Unternehmensleiter und Anlageberater verpflichtet werden, die Sparer und Investoren anhand eines zertifizierten Nachhaltigkeitsrating darüber $z u$ informieren, wieweit die in Betracht kommenden Anlageprodukte den Kriterien der Natur- und Sozialverträglichkeit genügen. Erst dadurch kann ethische Geldanlage mit der Zeit zur allgemeinen Norm werden.

Benachteiligung durch ihren eigenen Einblick in die Kosten des strittigen Produktionsverfahrens nachweisen können. 
Wenn diese rechtspolitischen Anliegen umgesetzt würden, wäre das ein wichtiger Beitrag für den Primat der Politik vor dem kapitalistischen Finanzkapital, für den Primat der Politik vor einem von Lobbyisten betriebenen Kapitalismus. Politik würde so aus ihrer Handlungsunfähigkeit befreit und geöffnet für Utopien und Visionen für den Aufbau einer zukunftsfähigen Marktwirtschaft, den Erhalt der Substanz unseres Natur-, Sozial- und Kulturkapitals und den Schutz der Gemeingüter.

Das entspräche ganz den von der UN im Jahr 2016 in der Agenda 2030 formulierten 17 Entwicklungsziele.

\section{Die Leitmotive der Agenda 2030 verlangen:}

- Armut und Hunger beenden und Ungleichheiten bekämpfen,

- Selbstbestimmung der Menschen stärken, Geschlechtergerechtigkeit und ein gutes und gesundes Leben für alle sichern,

- Wohlstand für alle fördern und Lebensweisen weltweit nachhaltig gestalten,

- ökologische Grenzen der Erde respektieren: Klimawandel bekämpfen, natürliche Lebensgrundlagen bewahren und nachhaltig nutzen,

- Menschenrechte schützen - Frieden und Rechtstaatlichkeit fördern und

- neue globale Partnerschaft aufbauen.

Bundeskanzlerin Merkel hat angesichts der Global Development Goals festgestellt, dass die bisherige Nachhaltigkeitsstrategie der Bundesregierung geändert werden muss und zur Umsetzung der Agenda völlig neu gestaltet werden muss. Damit wird ersichtlich, dass wir eine Änderung des Systems in dem aufgezeigten Sinne benötigen.

\section{Literatur}

Borgwardt, A. 2017. Impulse für die strategische Debatte in der Wissenschaft. In Netzwerk Exzellenz an deutschen Hochschulen. Berlin: Friedrich-Ebert-Stiftung.

Forschungsgruppe Ethisch-Ökologisches Rating HG. 2016. Systemänderung oder Kollaps unseres Planeten. Erkelenz: Erklärung der Forschungsgruppe Ethisch-Ökologisches Rating der Goethe-Universität Frankfurt - Arbeitskreis Wissenschaft. Federführung: Johannes Hoffmann.

Hennemann, G. 2008. Im Namen des Wettbewerbs. Süddeutsche Zeitung 11:17.

Hoffmann, J. Hrsg. 1992. Ethische Vernunft und technische Rationalität. Frankfurt: Verlag für Interkulturelle Kommunikation. 
Hoffmann, J. Hrsg. 1991. Das eine Menschenrecht für alle und die vielen Lebensformen. Band I: Begründung von Menschenrechten aus der Sicht unterschiedlicher Kulturen. Frankfurt: Verlag für Interkulturelle Kommunikation.

Hoffmann, J. Hrsg. 1994. Band II: Universale Menschenrechte im Widerspruch der Kulturen. Frankfurt. Band III: Die Vernunft in den Kulturen - Das Menschenrecht auf kultureigene Entwicklung. Frankfurt: Verlag für Interkulturelle Kommunikation.

Hoffmann, J. Hrsg. 1997. Irrationale Technikadaptation als Herausforderung an Ethik, Recht und Kultur. Interdisziplinäre Studien. Frankfurt: IKO, Verlag für Interkulturelle Kommunikation

Hoffmann, J. 1997. Zur Bedeutung der Kulturverträglichkeit. In Ethische Kriterien für die Bewertungen von Unternehmen. Frankfurt-Hohenheimer Leitfaden, Hrsg. J. Hoffmann, K. Ott, und G. Scherhorn, 263-291. Frankfurt.

Hoffmann, J., und G. Scherhorn, Hrsg. 2009. Eine Politik für Nachhaltigkeit. Neuordnung des Kapital- und Gütermärkte. Erkelenz: Altius-Verl.

Hoffmann, J., K. Ott, und G. Scherhorn, Hrsg. 1997. Ethische Kriterien für die Bewertung von Unternehmen - Frankfurt-Hohenheimer Leitfaden. Frankfurt a. M.: IKO - Verlag.

Homann, K., und M. Ungethüm. 2007. FAZ. Ethik des Wettbewerbs 143:11.

Peukert, H. 1976. Wissenschaftstheorie - Handlungstheorie - Fundamentale Theologie. Analysen zu Ansatz und Status theologischer Theoriebildung, 273. Düsseldorf: Suhrkamp.

Roche, P., J. Hoffmann, und W. Homolka, Hrsg. 1992. Ethische Geldanlagen. Kapital auf neuen Wegen. Frankfurt: Verlag IKO.

Scherhorn, G. 2005. Markt und Wettbewerb unter dem Nachhaltigkeitsziel. Zeit-schrift für Umweltpolitik \& Umweltrecht Beiträge zur rechts-, wirtschafts- und sozialwissenschaftlichen Umweltforschung 2:135-154.

Simmel, G. 1989. Philosophie des Geldes. Gesamtausgabe 6. Frankfurt: Suhrkamp.

Von Flotow, P. 1995. Geld, Wirtschaft und Gesellschaft. Georg Simmels Philosophie des Geldes. Frankfurt: Suhrkamp.

Johannes J. Hoffman, Prof. Dr., Professur für Moraltheologie, Sozialethik und Wirtschaftsethik am Fachbereich Katholische Theologie der Goethe-Universität in Frankfurt am Main; Diakon mit Zivilberuf der Diözese Limburg, Mitglied des Vorstandes von Theologie Interkulturell e. V.; Projektleiter der FG „Ethisch-Ökologisches Rating“ der Goethe-Universität; seit 2018 Mitglied der Forschungsgruppe Finanzen und Wirtschaft der Stiftung Weltethos an der Universität Tübingen.

www.ethisch-oekologisches-rating.org, http://blog.ethisch-oekologisches-rating.org/ 
Open Access Dieses Kapitel wird unter der Creative Commons Namensnennung 4.0 International Lizenz (http://creativecommons.org/licenses/by/4.0/deed.de) veröffentlicht, welche die Nutzung, Vervielfältigung, Bearbeitung, Verbreitung und Wiedergabe in jeglichem Medium und Format erlaubt, sofern Sie den/die ursprünglichen Autor(en) und die Quelle ordnungsgemäß nennen, einen Link zur Creative Commons Lizenz beifügen und angeben, ob Änderungen vorgenommen wurden.

Die in diesem Kapitel enthaltenen Bilder und sonstiges Drittmaterial unterliegen ebenfalls der genannten Creative Commons Lizenz, sofern sich aus der Abbildungslegende nichts anderes ergibt. Sofern das betreffende Material nicht unter der genannten Creative Commons Lizenz steht und die betreffende Handlung nicht nach gesetzlichen Vorschriften erlaubt ist, ist für die oben aufgeführten Weiterverwendungen des Materials die Einwilligung des jeweiligen Rechteinhabers einzuholen. 